\title{
Central Nervous System Manifestations in Diabetes Mellitus - A Review
}

GOUTAM KUMAR ACHERJYA, ${ }^{1}$ MD. MOSLEM UDDIN, ${ }^{2}$ M A JALIL CHOWDHURY, ${ }^{3}$ A V SRINIVASAN 4

\begin{abstract}
The impact of diabetes mellitus on the CNS (Central Nervous System) has gained attention only recently. Peripheral neuropathy has been the primary neuroscience focus of diabetes research. Contrary to some early impressions, however, the CNS is not spared by diabetes. Chronically, diabetes mellitus affects the CNS in several ways. Diabetes increases stroke risk and damage, overtreatment with insulin or oral agents can permanently damage the brain, and diabetes may increase the prevalence of seizure disorders. Diabetes changes brain transport, blood flow and metabolism, and may produce a chronic encephalopathy. Acutely, glycemic extremes cause coma, seizures, focal neurolclgical deficits, and impaired consciousness. The pathophysiological basis for these marked CNS abnormalities seen in hypoglycemia, hyperosmolar coma, and ketoacidosis are largely unknown.

Methods: This review was based on a search of Pubmed, the NCBI Database of systemic Reviews, and citation lists of relevant publications. Subject heading and key words used central nervous system, diabetes mellitus, stroke, encephalopathy and hypoglycaemia. Only articles in English were included.
\end{abstract}

Key Words: CNS, Diabetes Mellitus, Stroke, Encephalopathy, Hypoglycaemia.

\section{Introduction}

Diabetic neuropathy (DN), affects approximately $50 \%$ of the people who have diabetes mellitus. It is associated with significantly accelerated rates of several debilitating microvascular complications such as neuropathy, and macrovascular complications such as atherosclerosis and stroke.Diabetes mellitus, a common metabolic disease with a rising global prevalence, is associated with long-term complications of peripheral nervous system and the central nervous system. ${ }^{1,2}$ Atherosclerosis is common in smokers and those with high blood pressure and abnormal fat levels in the blood. It is commonly fatty deposits in arteries or hardening of arteries. It accounts for virtually $80 \%$ of all deaths among diabetic patients. It is reported that endothelial injury may be the initial event in the genesis of atherosclerosis, followed by platelet adhesion and aggregation at the site of injury. Prolonged exposure to

1. Junior Consultant (Medicine), Upazila Health Complex, Bagerpara, Jessore.

2. Senior Consultant (Medicine), 250 Bedded General Hospital, Jessore.

3. Professor and Chairman, Department of Medicine, BSMMU.

4. Emeritus Professor-The Tamil Nadu Dr.M.G.R. Medical University. Adjunct Professor-IIT Chennai. India.

Corresponding author: Dr. Goutam Kumar Acherjya, Junior Consultant (Medicine), Upazilla Health Complex, Bagerpara, Jessore. Mob: +8801711029790, e-mail: gacherjya@hotmail.com. hyperglycemia is now recognized a major factor in the pathogenesis of atherosclerosis in diabetes. Hyperglycemia induces a large number of alterations at the cellular level of vasculartissue that potentially accelerate the atherosclerotic process. Diabetesmellitus patients carry an increased risk two to four times greater forheart attack, stroke and other complications related to poorcirculation. ${ }^{3}$

Recent evidence suggests a key role for the brain in the control of energy metabolism, body fat content and glucose metabolism. Neuronal systems, which regulate energy intake, energy expenditure, and endogenous glucose production, sense and respond to input from hormonal and nutrientrelated signals that convey information regarding both body energy stores and current energy availability. In response to this input, adaptive changes occur that promote energy homeostasis and the maintenance of blood glucose levels in the normal range. Defects in this control system are implicated in the link between obesity and type 2 diabetes mellitus. Central nervous system may be considered as the conductor of an orchestra implicating many peripheral organs involved in these homeostatic processes. However, brain is mainly a glucose-dependent organ, which can be damaged by both hypoglycaemia and hyperglycaemia. Hypoglycaemiaunawareness is a major problem in clinical practice and is associated with an increased risk ofcoma. Stroke is another acute complication associated with diabetes 
mellitus, especially in elderly people, and control of glucose level in this emergency situation remains challenging. The prognosisof stroke is worse in diabetic patients and both the prevention management of at risk patients should be improved. Finally, chronic diabetic encephalopathies, which may lead to cognitive dysfunction and even dementia, are also recognized. They may result from recurrent hypoglycaemia and/or from chronic hyperglycaemia leading to cerebral vascular damages. Functional imaging is of interest to explore diabetes-associated cerebral abnormalities. Thus, the intimate relationship between brain and diabetes is increasingly acknowledged in both research and clinical practice. ${ }^{4}$

\section{Possible Mechanism:}

The mechanisms underlying neuronal apoptosis and CNS dysfunction are not clear. We believe that contributing factors are insulin deficiency with concomitant C-peptide deficiency, as well as hyperglycemia and possibly the aging process itself (Fig 1). ${ }^{5}$

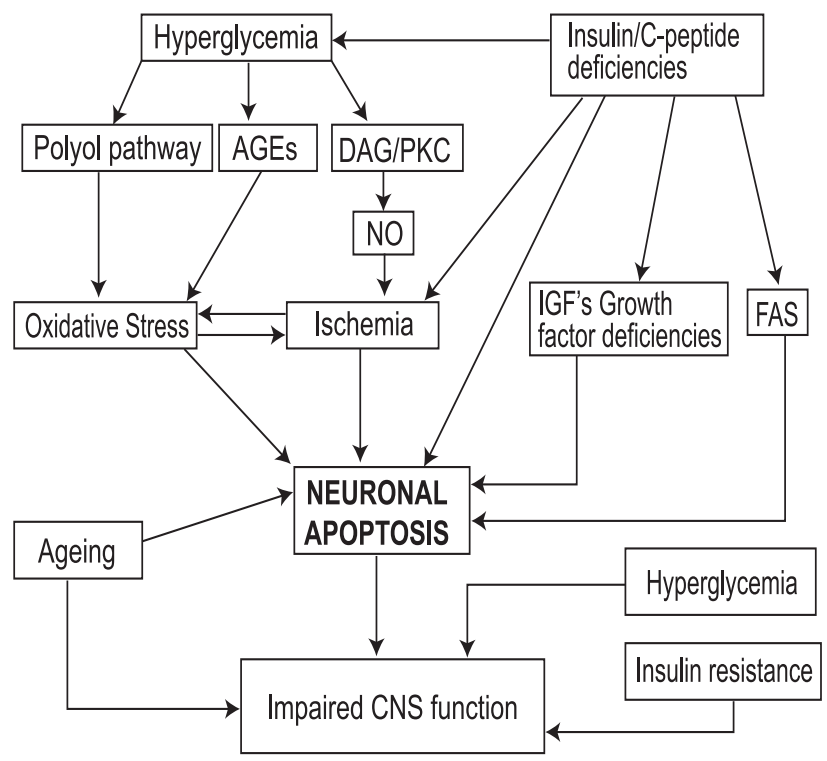

Fig 1: Possible pathways leading to CNS dysfunction in diabetes.

\section{CNS Complications in Type 1 Diabetic Patients}

Impairments in learning, memory, problem solving, andmental and motor speed are more common in type 1 diabetic patients than in the general population. The cognitive impairments can be severe in rare cases but is in most cases modest. The variation in severity and pattern of impairment may be due to the subtle nature of the defects and variation in the diabetic populations. Diabetic patients suffered from significantly more introversive symptoms than their healthy counterparts, especially with respect to somatic symptoms, sleeping disturbances, compulsions, and depressive mood. ${ }^{6}$

In terms of structural changes, several abnormalities have been described in type 1 diabetic patients, such as diffuse and local degenerative changes of cerebral cortex, neuronal loss, demyelination and gliosis, and infarction secondary to microangiopathy. Magnetic resonance imaging and computed tomography have shown that widened sulci and/ or enlarged lateral ventricles and increased occurrence of white matter hyperintensitiesare more pronounced in diabetic patients than in age-matched control subjects. ${ }^{7}$

\section{CNS Complications in Type 2 Diabetic Patients}

Neuropsychologic studies in type 2 diabetes patients have shown more consistent results compared to type 1 diabetespatients. Cognitive deficits and poor performance in abstract reasoning and complex psychomotor functioning occur in type 2 diabetes. Complex cognitive functions, as demonstrated by complex cognitive tasks requiring storage and retrieval of new information, are affected, whereas performances of less demanding tasks, such as immediate memory and simple reaction time, are not significantly altered. Recently, increased blood-brain barrier (BBB) permeability in type 2 diabetes was demonstrated by magnetic resonance imaging when intravenous administration of gadolinium diethylenetriaminepentaacetic acid (Gd-DTPA) was used to identify BBB permeability, suggesting that loss of BBB integrity may play a role in CNS dysfunction in diabetes. In the AllWales Research into Elderly (AWARE) Diabetes Study, elderly type 2 diabetic patients displayed significant excess of cognitive dysfunction associated with a poorer ability in diabetes self-care. However the current thought is that learning and memory dysfunction are more prominent in elderly type 2 diabetic subjects. ${ }^{8}$ The higher frequency of cerebral stroke in type 2 diabetes may be associated with cognitive deficits. Several studies have suggested that the cerebrovascular mortality rate is higher in patients with type 2 diabetes. The risk for developing stroke is increased 2- to 5-fold compared to nondiabetic control subjects. ${ }^{9}$

\section{Diabetes, stroke and control of glycaemia}

In DM, glycaemic control should be part of a global approach to vascular risk. The benefits of intervention to lower blood glucose in terms of microvascular health are well established but benefit on macrovascular, especially cerebrovascular, health has been less apparent. DM and hyperglycaemia per se are associated with poor cerebrovascular health, both in terms of stroke risk and outcome thereafter. A period of hyperglycaemia is common, with elevated blood glucose in the periinfarct period consistently linked with poor outcome 
in patients with and without DM. Hyperglycaemia can be either the cause or the result of severe brain injury. The association between poor glycaemic control and an unfavourable prognosis of patients with acute ischemic stroke is particularly evident in individuals with persistent hyperglycaemia, patients without a known history of DM, and subjects with cortical infarction. In acute stroke, theoretical data suggest intervention to lower markedly elevated blood glucose may be of benefit, especially if thrombolysis is administered. ${ }^{10}$

Reducing the excess cerebrovascular burden in patients with type 2 DM remains a major therapeutic challenge, especially with respect to the high risk of recurrent events. Targeting the traditional metabolic risk factors of hypertension, dyslipidaemia, and hyperglycaemia has failed to remove this excess risk, and agents targeting thrombotic risk (i.e., antiplatelet and anticoagulant drugs) remain poorly studied in the context of stroke in DM. Overall, there is a need for rigorous evaluations of new therapeutic approaches in both primary and secondary prevention of stroke as well as management of acute stroke in patients with type $2 \mathrm{DM}$ increased risk of the patient developing hypoglycaemia, which may be also deleterious for the brain. ${ }^{11}$

\section{Hypoglycaemia and brain}

Most patients treated with insulin, and some on excessive doses of sulphonylureas, experience hypoglycaemia at some time. Patients may experience symptoms of hypoglycaemia when the blood glucose is less than $3 \mathrm{mmol} / \mathrm{l}$ although some who have lost their alerting symptoms may pass below this threshold without warning. The onset of neuroglycopenic features is however, associated with diminished cognitive function which may lessen the capacity to take corrective measures. Untreated hypoglycaemia can then progress to restless or even violent behavior, unconsciousness, seizures, and (rarely) reversible hemiplegia. The cause of the very rare "dead in bed" syndrome described in some young type 1 patients is not known and only speculatively associated with hypoglycaemia and perhaps hypokalaemia as well. ${ }^{12}$

\section{Diabetic encephalopathy}

Diabetic encephalopathy is characterized by neurophysiological impairments, structural changes, and cognitive deficits. Diabetic encephalopathies are now accepted complications of DM. They appear to differ in type 1 and type $2 \mathrm{DM}$ as to underlying mechanisms and the nature of resulting cognitive deficits.According to epidemiological data, the increased incidence of Alzheimer's disease in type
$2 \mathrm{DM}$ is associated with insulin resistance, hyperinsulinaemia and hyperglycaemia, and commonly accompanying attributes such as hypercholesterolemia, hypertension and (abdominal) obesity. The pathobiology of accumulation of beta-amyloid and tau protein, the hallmarks of Alzheimer's disease, has been demonstrated in experimental data. Type 1 diabetic encephalopathy is likely to increase as a result of the global increase in the incidence of type $1 \mathrm{DM}$ and its occurrence in increasingly younger patients may represent an alarming hazard. ${ }^{13}$

\section{Conclusion}

CNS may also be exposed to acute damages in case of eithersevere hypoglycaemia or hyperglycaemia, but also may be exposed to various chronic encephalopathies, some of them mimicking Alzheimer disease. Substantial evidence collected from clinical data and experimental studies has indicated that CNS is not spared from diabetes complications. Impairments in CNS function are well documented in both type 1 and type 2 diabetic patients as well as in various animal models of diabetes, in terms of alterations in cognition, neuropsychology, neurobehavior, electrophysiology, structure, neurochemistryand apoptotic activities.Specific prevention and treatment strategies should be evaluated and implemented in patients to reduce the increasingly recognized burden associated to diabetes-related CNS disorders.

\section{Conflict of interest: None.}

\section{References}

1. Plum F: The neurologic complications of diabetes mellitus. In Diabetes. Williams RH, Ed. New York, Hoeber 1960:60222.

2. Chen D, Huang H, Xing Y, Liu Y, Xu Y, et al. A New Vanadium Complex Improves the Spatial Learning and Memory by Activation of Caveolin-MAPK-CREB Pathway in Diabetic Mice. J Diabetes Metab 2011;2:114.

3. Aronson D, Rayfield EJ. How hyperglycemia promotes atherosclerosis:molecular mechanisms. CardiovascDiabetol 2002;1:1.

4. Diabetic Neuropathy. Available from: http:// diabetesinformationhub.com /DiabetesComplications DiabeticNeuropathy.php. Accessed on 15.09.2016.

5. Gerozissis K., Rouch C., Lemierre S., Nicolaidis, S., and Orosco,M. Apotential role of central insulin in learning and memoryrelated to feeding. Cell. Mol. Neurobiol. 2001;21:389-401.

6. McCarthy A. M., Lindgren S., Mengeling M. A., Tsalikian E., and Engvall J. C. Effects of diabetes on learning in children. Pediatrics 2002:109. 
7. Araki, Y., Nomura, M., Tanaka, H., Yamamoto, H., Yamamoto,T., Tsukaguchi, I., and Nakamura, H. MRI of the brain in diabetes mellitus. Neuroradiology 1994;36:101-103.

8. Ryan, C. M., and Geckle, M. Why is learning and memorydysfunction in Type 2 diabetes limited to older adults? DiabetesMetab. Res. Rev. 2000;16:308-315.

9. Manson, J. E., Colditz, G. A., Stampfer, M. J., Willett, W. C.,Krolewski, A. S., Rosner, B., Arky, R. A., Speizer, F. E., andHennekens, C. H. A prospective study of maturity-onset diabetes mellitus and risk of coronary heart disease and stroke inwomen. Arch. Intern. Med. 1991;151:1141-1147.
10. Quinn TJ, Dawson J, Walters MR. Sugar and Stroke: Cerebrovascular disease and bloodglucose control. Cardiovasc Ther 2010;29(6):e31-42.

11. Sander D, Kearney MT. Reducing the risk of stroke in type 2diabetes: pathophysiological and therapeutic perspectives. J Neurol 2009;256:1603-19.

12. Frier B, Fisher M, eds. Hyperglycaemia and diabetes. Clinicaland physiological aspects. London: Edward Arnold, 1993.

13. Sima AA. Encephalopathies: the emerging diabetic complications. Acta Diabetol. 2010;47(4):279-93. 